\title{
Endoscopic Removal of Foreign Body in Upper Gastrointestinal Tract in Dogs: Success Rate and Complications
}

\author{
Franco Metzker Poggiani' ${ }^{1}$, Rodrigo Pereira da Costa Duarte², Marcelo Ismar Silva Santana ${ }^{2} \&$ Paula Diniz Galera ${ }^{2}$
}

\begin{abstract}
Background: Dogs and cats with acute signs of choking, retching, cough, vomiting, regurgitation, hypersalivation, dysphagia and odynophagia should have the presence of a gastrointestinal foreign body (FB) as part of their differential diagnosis, where it is a frequent condition in the care of small animals. Most objects lodged in the esophagus, stomach, and proximal duodenum can be removed by upper digestive endoscopy, a curative, little invasive procedure. The objective of our study was to evaluate the physical aspects and location of esophageal and gastric FBs observed in 88 dogs and the age and breed of the affected animals, and to determine the success rate and eventual complications associated with the procedure as well. Materials, Methods \& Results: Eighty-eight cases of dogs, males and females of varying ages and breeds, submitted to upper digestive endoscopy were selected because of suspicion of esophageal or gastric FBs. The endoscopic procedure aimed at confirming the diagnosis, whether or not followed by endoscopic removal of these objects. Prior to endoscopy, the animals had laboratory tests (blood count and serum biochemistry) and subsequently to the anesthetic protocols of choice for each case. Data including breed, age, type of constituent material and anatomical location of the FB, endoscopic procedure success rate and complications were recorded and descriptively evaluated. Of the 88 dogs evaluated, $60 \%$ (n $=53)$ were male and $40 \%(n=35)$ female. According to the breed of the animals, 55\% $(n=49)$ were small-breed dogs, $29 \%(\mathrm{n}=25)$ large-breed dogs, and $8 \%(\mathrm{n}=7)$ medium-breed dogs, and $8 \%$ were of mixed breed dogs, which could assume various sizes. Shih tzus accounted for $18 \%(n=16)$ of the animals, Lhasa apso $8 \%(n=7)$ and mixed breed $8 \%(n$ $=7$ ), where these were the most frequently affected breeds. Regarding age, animals 1 to 5 years old represented $66 \%$ (n $=58)$ of the patients, and those 6 to 10 years old accounted for $20 \%(\mathrm{n}=18)$, while $11 \%$ of the dogs were over 10 years old $(\mathrm{n}=10)$. Two animals $(3 \%)$ had no information about their ages. Pieces of cloth were the most frequently found FBs, representing $20 \%(\mathrm{n}=20)$, followed by animal bones $(19 \%)$ and fruit pits $(10 \%)$. As for location, $78 \%(\mathrm{n}=69)$ of the FBs were located in the stomach and $22 \%(\mathrm{n}=19)$ in the esophagus. The success rate of endoscopic FB removal in this study was $83 \%(n=73)$. In $76 \%(n=67)$ of the animals, there were no complications due to the presence of FB in the upper gastrointestinal tract. The most frequent complications were esophageal ulcerations $(n=7)$ and inability to move the FB $(\mathrm{n}=5)$ and adherences $(\mathrm{n}=4)$.

Discussion: The results showed that small-breed dogs, especially Shih tzus and Yorkshires, represented a larger number of cases, probably due to their popularity in Brazil, where the study was conducted. Males were more prevalent than females, and the most affected age was between 1 and 5 years, with emphasis on younger animals. There were more gastric FB cases compared to esophageal FB cases, which was related to the interval between ingestion of the object and veterinary care. Although not the most prevalent FB, the high rate of mango pits can be explained by the vast number of mango trees in the Federal District. There were few complications compared to the success of cases, indicating that endoscopy is the procedure of choice for the diagnosis and removal of FBs from the gastrointestinal tract.
\end{abstract}

Keywords: endoscopy, foreign body, stomach, esophagus, dogs. 


\section{INTRODUCTION}

The presence of a foreign body (FB) in the upper gastrointestinal tract is frequent in dogs and cats [20], where it is considered an emergency if located in the esophagus. Animals with acute signs of choking, retching, cough, vomiting, regurgitation, ptyalism, dysphagia and odynophagia should have the presence of a gastrointestinal FB included in their differential diagnosis [21].

Bones are the most commonly reported gastrointestinal FBs, although snacks, balls, toys, hooks, pieces of wood and needles have also been reported $[1,6,7,16,21]$. Although rare, possible complications associated with endoscopic removal of FBs include perforation, hemorrhage, malfunctions in moving the FB making it more difficult to remove, breathing problems related to esophageal perforation with possible tracheal involvement, and the formation of stenosis when there is damage to the esophageal mucosa [20].

Most objects lodged in the esophagus, stomach and proximal duodenum can be removed endoscopically. It is recommended that a curative and less invasive procedure such as upper digestive endoscopy be performed immediately whenever possible, avoiding progressive worsening of the esophageal mucosa and consequently necrosis and other secondary complications $[15,18]$.

Advantages of the procedure include low invasiveness, high success rate, and shorter performance time compared to surgical procedures [6]. Accordingly, the objective was to evaluate the physical aspects and location of esophageal and gastric FBs observed in 88 dogs and the ages and breeds of the affected animals, and to determine the success rate and complications associated with the procedure as well.

\section{MATERIALS AND METHODS}

\section{Animals}

We selected 88 cases of dogs, males and females of varying ages and breeds, that were submitted to upper digestive endoscopy because of suspicion of esophageal or gastric FBs. The endoscopic procedure aimed at confirming the diagnosis, whether or not followed by endoscopic removal of these objects. Prior to the procedure, the animals underwent laboratory tests (blood count and serum biochemistry) and, subsequently, the anesthetic protocols of choice for each case.
Endoscopic procedures

In the endoscopic procedures, we used a flexible videogastroscope (Olympus GIF-100) ${ }^{1}$ with a 100 $\mathrm{mm}$ long and $9.8 \mathrm{~mm}$ diameter insertion tube with a 2.2-mm working channel and a flexible videocolonoscope (Olympus GIF-100) ${ }^{2}$ with a $130 \mathrm{~mm}$ long and $13.0 \mathrm{~mm}$ diameter insertion tube, with 2.2-mm working channel. The choice of one or the other endoscope for the procedure was made according to the size of the patient, and the videocolonoscope was used for large and very large patients to allow access and adequate visualization of the pyloric antrum. For removal of the FB, we used endoscopic grasping forceps ${ }^{3}$ and also of the handle type. Images were obtained and recorded using a video capture card $\left(\text { Zscan }^{\circledR}\right)^{4}$.

The endoscopy technique was according to Guilford [5], by introducing the endoscope into the oral cavity and directing it to the dorsal-caudal epiglottis region. After gas insufflation by the equipment, the pharyngoesophageal junction was crossed, and it was possible to visualize the esophageal lumen. The entire esophageal length was inspected down to the cardiac ostium. After passage through the cardiac ostium, it was possible to observe the mucosal pool and folds of the gastric body. Subsequently, after stomach insufflation, the gastric fundus was inspected by an endoscopic retroflexion maneuver, followed by observation of the antrum and pylorus.

After locating the object in the esophageal or gastric lumen, the endoscopic forceps best suited for that type of material were introduced through the working channel of the equipment. The object was then seized and the endoscope retracted toward the oral cavity, along with the FB to be removed (Figures 1 and 2). The endoscopic procedure was considered successful in the complete removal of the FB orally without the need for surgical intervention or complications resulting from the procedure. Endoscopy was deemed a failure when it was not feasible to remove the FB or when it was necessary to push it into the stomach for later gastrotomy.

Data including breed, age, type of constituent material of FB, anatomical location, endoscopic procedure success rate and complications were recorded (Table 1 ), and descriptive analysis of the data was performed.

\section{RESULTS}

Of the 88 dogs evaluated, $60 \%(n=53)$ were male and $40 \%(n=35)$ female. According to the breed of animals, 55\% ( $n=49)$ were small-breed dogs, $29 \%$ 
$(\mathrm{n}=25)$ large-breed dogs, and 8\% $(\mathrm{n}=7)$ medium-breed dogs, and $8 \%$ were of mixed breed, which could assume various sizes, but had no documented data. Among the most represented breeds in this study, Shih tzus accounted for $18 \%(\mathrm{n}=16)$ of the animals, Lhasa apso $8 \%(\mathrm{n}=7)$, mixed breed $8 \%(\mathrm{n}=7)$, poodles $7 \%(\mathrm{n}=$ $6)$, Yorkshires 6\% $(n=5)$ and Rottweilers 5\% $(n=4)$. Regarding age, the animals evaluated were between 1 and 13 years old, and those 1 to 5 years old represented $66 \%(\mathrm{n}=58)$ of the patients, those 6 to 10 years old $20 \%(\mathrm{n}=18)$ and those over 10 years old $11 \%(\mathrm{n}=$ 10). The ages of two animals (3\%) were not recorded.

Pieces of cloth were the most frequently found FBs, representing 20\% $(n=20)$, followed by animal bones $(19 \%)$, with $14 \%(\mathrm{n}=12)$ being pork and $5 \%$ $(\mathrm{n}=4)$ chicken, and fruit pits $(10 \%)$, with $7 \%(\mathrm{n}=$ 6) being mango and $3 \%(n=3)$ peach (Figure 3$)$. As for location, $78 \%(n=69)$ of the FBs were located in the stomach and $22 \%(\mathrm{n}=19)$ in the esophagus. With regard to gastric $\mathrm{FBs}, 42 \%(\mathrm{n}=29)$ were only in the antrum, 29\% ( $n=20)$ only in the body and 9\% $(n=6)$ only in the fundus. Gastric FBs were commonly observed in more than one region of the organ (Graph 1). Among the esophageal FBs, 53\% $(n=10)$ were located in the esophageal hiatus, $31 \%(n=6)$ at the heart base height, $11 \%(\mathrm{n}=2)$ in the cervical esophagus and $5 \%$ $(n=1)$ in the chest entrance (Graph 2). FB obstruction of the esophageal lumen was complete in most cases.

The success rate of endoscopic FB removal in this study was $83 \%(n=73), 89 \%$ (61 of 69 cases) in gastric removal and 63\% (12 of 19 cases) in esophageal removal. In general, after endoscopic FB removal, the mucosa was normal in appearance. However, in some cases it was possible to observe mild to moderate enanthem.

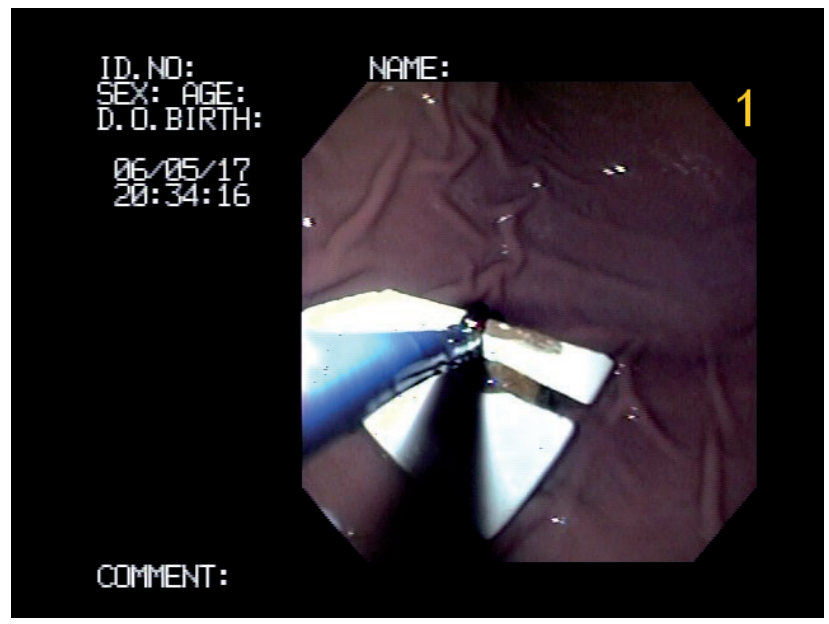

Figure 1. Piece of tile in the stomach removed by endoscopy.
Of the $11 \%(\mathrm{n}=8)$ of cases in which endoscopic removal of a gastric FB was not possible, $7 \%$ (n $=5$ ) of the animals underwent gastrotomy and $4 \%$ (n $=3)$ gastrotomy and enterotomy. Of the $37 \%(n=7)$ of unsuccessful cases of endoscopic withdrawal of an esophageal FB, $21 \%(n=4)$ of the animals underwent esophagotomy and $16 \%(\mathrm{n}=3)$ had the FB pushed into the stomach, which led to a change in the surgical intervention from thoracic esophagotomy to gastrotomy.

In $76 \%(n=67)$ of the animals, there were no complications due to the presence of an FB in the upper gastrointestinal tract. Among those with complications, the most frequently found were esophageal ulcerations $(n=7)$, the inability to move the FB $(n=$ 5) and adherences $(n=4)$ [Graph 3].

\section{DISCUSSION}

The improvement of flexible fiber endoscopes for video endoscopes, and the development of FB-grasping forceps, basket forceps, and polypectomy handle have made endoscopic removal the procedure of choice for the removal of esophageal and gastric FBs [19]. A technique involving the use of fluoroscopy-guided forceps has been described, however, little scientific information has been published on the subject or the long-term clinical evolution of the animals involved in such studies [12]. Due to their versatility, the most popular endoscopes in small animal practice are gastroscopes, which have four-way deflection of the tip. A gastroscope less than $9 \mathrm{~mm}$ in diameter and at least $130 \mathrm{~cm}$ long is suitable in most dogs for upper and lower digestive endoscopy [19].

There are diverging opinions on the ideal type of endoscope for performing esophageal FB removal. While some authors have found that the use of flexible

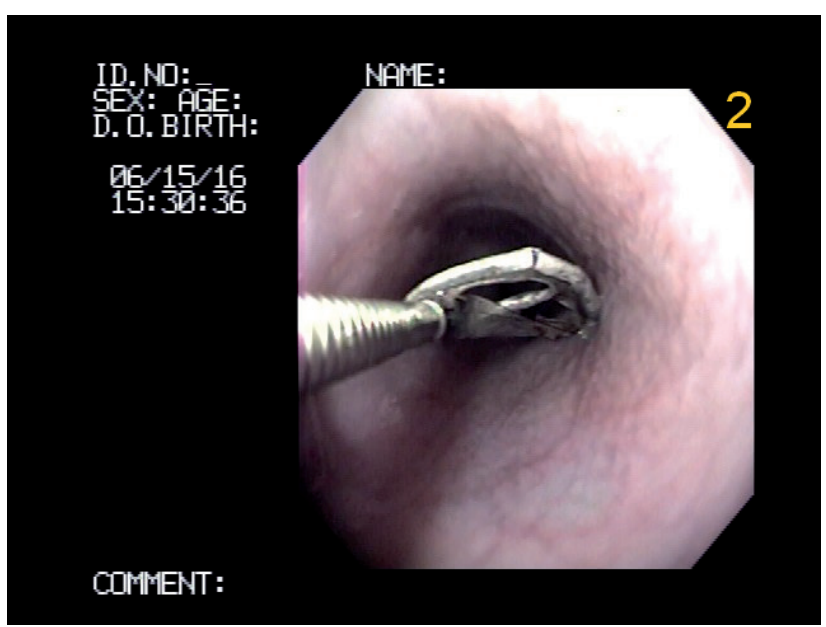

Figure 2. Plastic bottle cap seal in the esophagus removed by endoscopy. 


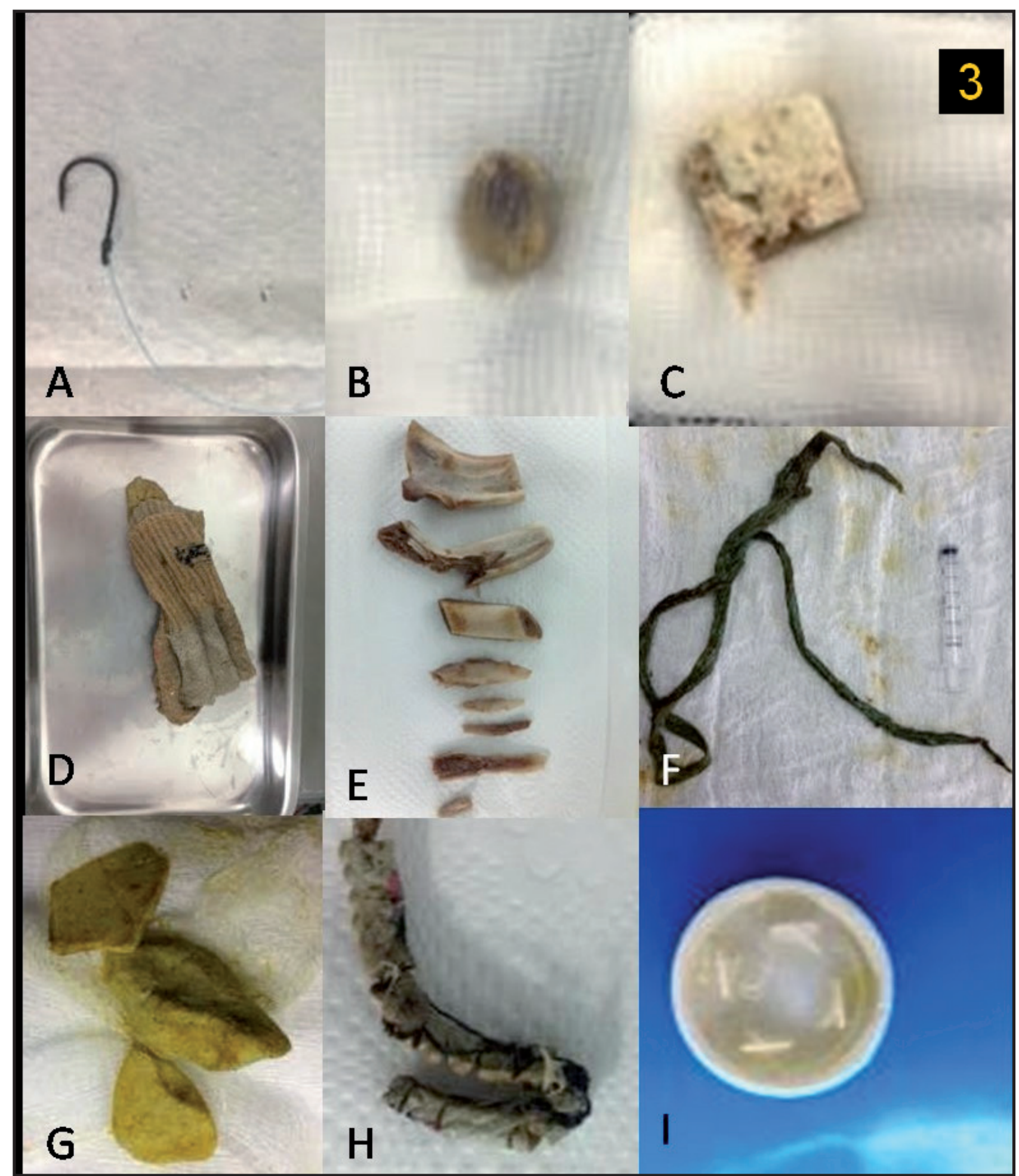

Figure 3. Main gastric and esophageal foreign bodies removed by endoscopy of the upper digestive tract. A- fishing hook. B- fruit pit (mango). C- synthetic leather bone. D- socks. E- bones. F- plastic bag. G- rocks. H- hard plastic (toy). I- milk carton lid.

endoscopes is the most appropriate way to evaluate the FB and esophageal mucosa integrity [17], others emphasize the advantage of using the largest diameter rigid endoscope to remove esophageal FBs when removal by flexible endoscopy becomes difficult or even impossible. This is due to the ability of rigid endoscopy to allow greater distension of the esophageal wall immediately cranial to the FB [8] and, the use of lateral endoscopic forceps. As these forceps does not need to be inserted through the endoscope's working channel, they can have a larger diameter, be more robust and have greater amplitude of object grasping, which could increase the likelihood of successful endoscopic removal of an esophageal FB. 
Tabela 1. Description of the sex, breed, age, foreign body, localization in the affected organ and the FB removal procedure in 88 dogs between 2013 and 2019.

\begin{tabular}{|c|c|c|c|c|c|c|c|}
\hline Name & Sex & Breed & $\begin{array}{l}\text { Age } \\
\text { (years) }\end{array}$ & Foreign Body & Organ & Organ Region & Removal \\
\hline 1 & Male & Border Collie & 6 & Ring and rocks & Stomach & Body and Fundus & Endoscopic \\
\hline 2 & Male & Bulldog & 1 & Mango pit & Stomach & Antrum and Fundus & Endoscopic \\
\hline 3 & Male & Bulldog & 5 & Ball of string & Stomach & Body & Endoscopic \\
\hline 4 & Male & Bulldog & 1 & Peach pit & Stomach & Antrum & Endoscopic \\
\hline 5 & Male & Bulldog & 1 & Mango pit & Stomach & Antrum and Fundus & Endoscopic \\
\hline 6 & Male & Bulldog & 1 & Toy & Stomach & Antrum & Endoscopic \\
\hline 7 & Female & Campeiro Bulldog & 3 & Rubber ball & Stomach & Body and Fundus & Gastrotomy \\
\hline 8 & Male & Bull Terrier & 3 & Pieces of cloth & Stomach & Antrum & Endoscopic \\
\hline 9 & Female & Bull Terrier & 8 & Mango pit & Stomach & Fundus & Endoscopic \\
\hline 10 & Male & Bull Terrier & 4 & Hard plastic, wire & Stomach & Antrum & Endoscopic \\
\hline 11 & Female & Bull Terrier & 3 & Pieces of cloth & Stomach & Antrum & Endoscopic \\
\hline 12 & Female & Chihuahua & 3 & Peach pit & Stomach & Antrum & Endoscopic \\
\hline 13 & Male & Cocker Spaniel & 4 & Wine cork & Stomach & Body and Antrum & Endoscopic \\
\hline 14 & Male & Golden & 1 & Rocks & Stomach & Antrum & Endoscopic \\
\hline 15 & Male & Golden & 1 & Electricity wire & Stomach & Body & Endoscopic \\
\hline 16 & Male & Golden & 3 & Pieces of cloth & Stomach & Body & Endoscopic \\
\hline 17 & Male & Siberian Husky & 13 & Metallic spoon & Stomach & Antrum & Endoscopic \\
\hline 18 & Male & Siberian Husky & 7 & Clothespin & Stomach & Antrum & Endoscopic \\
\hline 19 & Female & Siberian Husky & 1 & Pieces of cloth & Stomach & $\begin{array}{l}\text { Antrum and Pyloric } \\
\text { canal }\end{array}$ & $\begin{array}{l}\text { Gastrotomy and } \\
\text { enterotomy }\end{array}$ \\
\hline 20 & Male & Labrador & 6 & Cloth and sticking plaster & Stomach & Body & Endoscopic \\
\hline 21 & Female & Labrador & 3 & Mango pit & Stomach & Fundus & Gastrotomy \\
\hline 22 & Female & Lhasa Apso & 1 & Chewing treat & Stomach & Body & Endoscopic \\
\hline 23 & Male & Lhasa Apso & 3 & Ball of string & Stomach & Body & Endoscopic \\
\hline 24 & Female & Lhasa Apso & 7 & Cloth, hair & Stomach & Fundus & Endoscopic \\
\hline 25 & Female & Lhasa Apso & 9 & Wood, wire, cloth & Stomach & Antrum & Gastrotomy \\
\hline 26 & Male & Lhasa Apso & 2 & Chicken bone & Esophagus & Esophageal hiatus & Endoscopic \\
\hline 27 & Female & Lhasa Apso & 4 & Sock & Stomach & Body and Antrum & Endoscopic \\
\hline 28 & Male & Lhasa Apso & 3 & Chicken bone & Stomach & Body & Endoscopic \\
\hline 29 & Male & Pomeranian & 1 & Chicken bone & Esophagus & Thoracic inlet & Endoscopic \\
\hline 30 & Female & Maltese & 4 & Pieces of sandals & Stomach & Body and Antrum & Endoscopic \\
\hline 31 & Male & German Shepherd & 2 & Sock & Stomach & Body & Endoscopic \\
\hline 32 & Male & German Shepherd & 8 & Pieces of cloth & Stomach & $\begin{array}{l}\text { Body, Antrum and } \\
\text { Pyloric canal }\end{array}$ & $\begin{array}{c}\text { Gastrotomy and } \\
\text { enterotomy }\end{array}$ \\
\hline 33 & Male & German Shepherd & 6 & Pieces of cloth & Stomach & $\begin{array}{l}\text { Antrum and Pyloric } \\
\text { canal }\end{array}$ & $\begin{array}{c}\text { Gastrotomy and } \\
\text { enterotomy }\end{array}$ \\
\hline 34 & Male & Malinois & 8 & Cloth and weed & Stomach & Body & Endoscopic \\
\hline 35 & Male & Malinois & 8 & Plastic bag with numbing drug & Stomach & Body & Endoscopic \\
\hline 36 & Female & Pinscher & 2 & Rubber part of a can & Stomach & Antrum & Endoscopic \\
\hline 37 & Male & Pinscher & 1 & Pig bone & Esophagus & Esophageal hiatus & Endoscopic \\
\hline 38 & Male & Pinscher & 2 & Fish scale & Esophagus & Cardiac base & Endoscopic \\
\hline 39 & Male & Pinscher & 2 & Marble & Stomach & Antrum & Endoscopic \\
\hline 40 & Female & Pitbull & 11 & Rocks & Stomach & Antrum & Endoscopic \\
\hline 41 & Female & Pitbull & 11 & Corn cob & Stomach & Antrum & Endoscopic \\
\hline 42 & Female & Poodle & - & Cloth, hair & Stomach & Antrum & Endoscopic \\
\hline
\end{tabular}


Tabela 1. Description of the sex, breed, age, foreign body, localization in the affected organ and the FB removal procedure in 88 dogs between 2013 and 2019.

\begin{tabular}{|c|c|c|c|c|c|c|c|}
\hline 43 & Female & Poodle & 13 & Hair, pieces of hard plastic & Stomach & Antrum & Endoscopic \\
\hline 44 & Female & Poodle & 2 & Plastic bag & Stomach & Antrum & Endoscopic \\
\hline 45 & Female & Poodle & 2 & Cloth, string & Stomach & Body & Endoscopic \\
\hline 46 & Male & Poodle & 3 & Plastic bag & Stomach & $\begin{array}{l}\text { Antrum, Pyloric canal, } \\
\text { duodenum }\end{array}$ & Endoscopic \\
\hline 47 & Male & Poodle & 12 & Pieces of cloth, wire and wood & Stomach & Body and Fundus & Endoscopic \\
\hline 48 & Female & Rottweiler & 11 & Chewing treat & Esophagus & Esophageal hiatus & Endoscopic \\
\hline 49 & Male & Rottweiler & 6 & String & Stomach & Body & Endoscopic \\
\hline 50 & Female & Rottweiler & 3 & Rubber pieces & Stomach & Body & Endoscopic \\
\hline 51 & Male & Rottweiler & 11 & Pig bone & Esophagus & Esophageal hiatus & Endoscopic \\
\hline 52 & Female & Schipperke & 3 & Pig bone & Esophagus & Esophageal hiatus & Gastrotomy \\
\hline 53 & Male & Shih Tzu & 3 & Chicken bone & Esophagus & Cardiac base & Esophagotomy \\
\hline 54 & Male & Shih Tzu & 1 & Sock & Stomach & Antrum & Endoscopic \\
\hline 55 & Female & Shih Tzu & 2 & Metallic part of clothespin & Stomach & Antrum & Endoscopic \\
\hline 56 & Male & Shih Tzu & 12 & Thread & Stomach & Pyloric canal & Endoscopic \\
\hline 57 & Female & Shih Tzu & 1 & Trichobezoar & Stomach & Body & Endoscopic \\
\hline 58 & Male & Shih Tzu & 2 & Mango pit & Stomach & Body & Gastrotomy \\
\hline 59 & Female & Shih Tzu & 1 & Pieces of hard plastic & Stomach & Antrum & Endoscopic \\
\hline 60 & Male & Shih Tzu & 5 & Pig bone & Esophagus & Esophageal hiatus & Gastrotomy \\
\hline 61 & Male & Shih Tzu & 6 & Fish hook & Stomach & Antrum & Endoscopic \\
\hline 62 & Male & Shih Tzu & 5 & Plastic milk packaging lid & Stomach & Antrum & Endoscopic \\
\hline 63 & Female & Shih Tzu & 1 & Rubber toy & Stomach & Fundus & Endoscopic \\
\hline 64 & Male & Shih Tzu & 2 & Sock & Stomach & Fundus & Endoscopic \\
\hline 65 & Male & Shih Tzu & 3 & Pig bone & Esophagus & Esophageal hiatus & Endoscopic \\
\hline 66 & Male & Shih Tzu & 1 & Sock & Stomach & Antrum & Endoscopic \\
\hline 67 & Male & Shih Tzu & 2 & Clothespin & Stomach & Antrum & Endoscopic \\
\hline 68 & Male & Shih Tzu & 12 & Thread & Stomach & Pyloric canal & Endoscopic \\
\hline 69 & Male & Swergspitz & 1 & Chewing treat & Esophagus & Esophageal hiatus & Endoscopic \\
\hline 70 & Female & Swergspitz & 3 & Pig bone & Esophagus & Esophageal hiatus & Endoscopic \\
\hline 71 & Male & Mixed Breed & 1 & Metallic clamp & Stomach & Body & Endoscopic \\
\hline 72 & Male & Mixed Breed & - & Fish hook & Esophagus & Cardiac base & Endoscopic \\
\hline 73 & Female & Mixed Breed & 2 & Rubber toy & Stomach & Antrum & Endoscopic \\
\hline 74 & Male & Mixed Breed & 10 & Cloth, stick & Stomach & Body & Endoscopic \\
\hline 75 & Female & Mixed Breed & 3 & Pig bone & Stomach & Fundus & Endoscopic \\
\hline 76 & Female & Mixed Breed & 4 & Pig bone & Esophagus & Esophageal hiatus & Gastrotomy \\
\hline 77 & Female & Mixed Breed & 10 & Toothpick and pieces of cloth & Stomach & Body & Endoscopic \\
\hline 78 & Male & Teckel & 6 & Plastic & Stomach & Antrum & Gastrotomy \\
\hline 79 & Female & Teckel & 7 & Peach pit & Stomach & Antrum & Endoscopic \\
\hline 80 & Female & Teckel & 6 & Cloth, hard plastic & Stomach & Antrum & Endoscopic \\
\hline 81 & Male & Teckel & 6 & Mango pit & Stomach & Antrum & Endoscopic \\
\hline 82 & Female & Teckel & 3 & Pig bone & Esophagus & Cardiac base & Esophagotomy \\
\hline 83 & Male & West Terrier & 2 & Pig bone & Esophagus & Cardiac base & Esophagotomy \\
\hline 84 & Male & Yorkshire & 1 & Pig bone & Esophagus & Cervical & Endoscopic \\
\hline 85 & Male & Yorkshire & 2 & Fruits & Stomach & Body & Endoscopic \\
\hline 86 & Female & Yorkshire & 11 & Fish hook & Esophagus & Cardiac base & Esophagotomy \\
\hline 87 & Female & Yorkshire & 1 & Pig bone & Esophagus & Cervical & Endoscopic \\
\hline 88 & Male & Yorkshire & 2 & Mango & Stomach & Body & Endoscopic \\
\hline
\end{tabular}




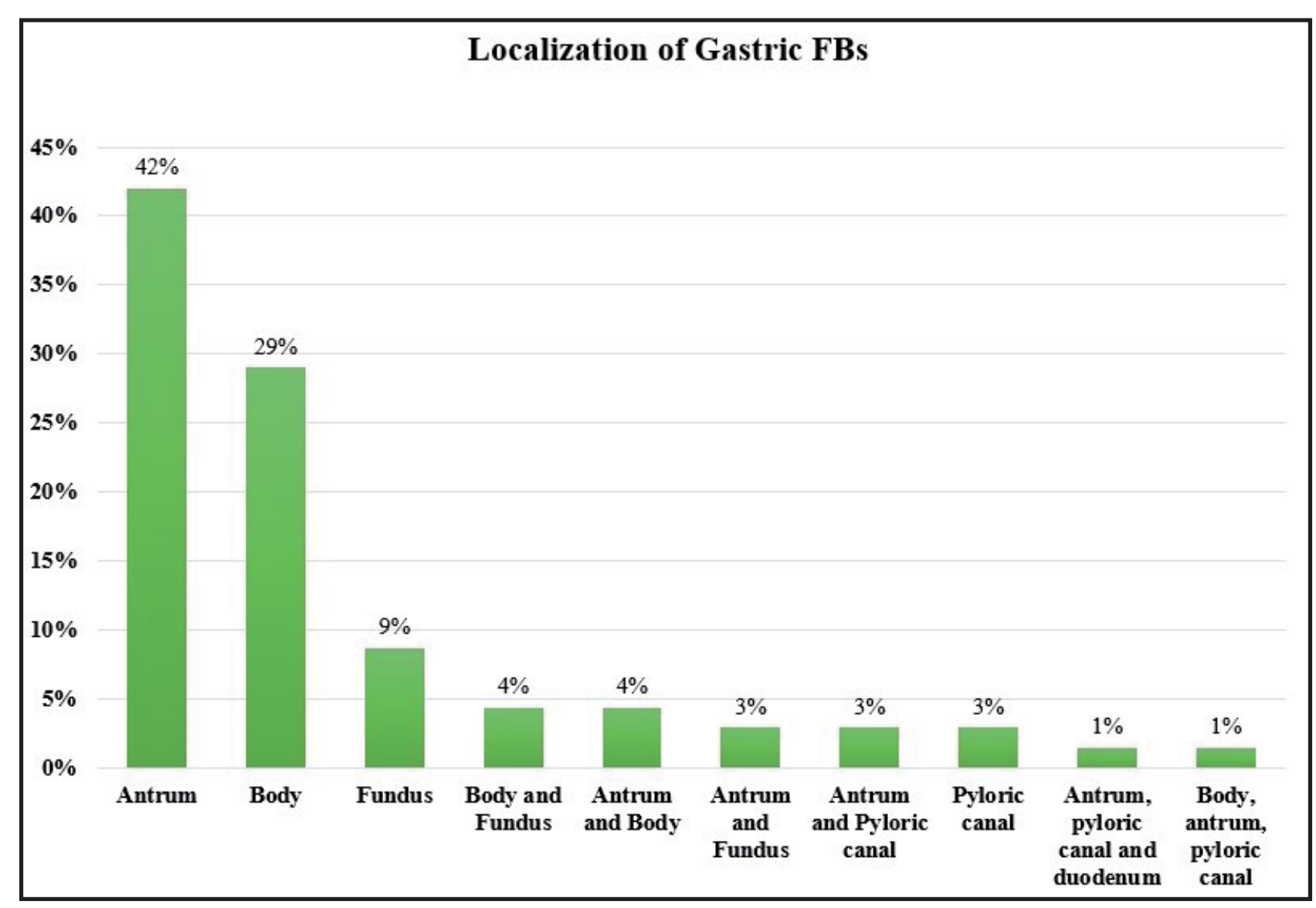

Graph 1. Localization of gastric FBs removed from 69 dogs between 2013 and 2019.

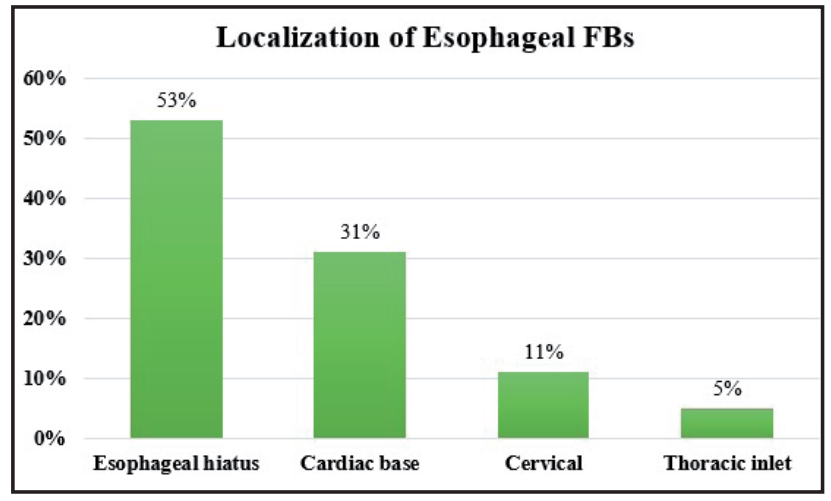

Graph 2. Localization of esophageal FBs removed from 19 dogs between 2013 and 2019.

Gastrointestinal FBs are a common diagnosis among dogs that are brought in for emergency veterinary care and yet pose a challenge [7]. Most (78\%) of the FBs in the present study were located in the stomach, unlike previous studies that refer to the esophagus [6] or the small intestine [8] of dogs as being the most common site where FBs are lodged.

Removal of a gastrointestinal FB is the best example of the therapeutic potential of endoscopy [14]. In this study, it was found that endoscopy was successful; that is, the FB was orally removed in $83 \%$ of cases, corroborating the literature data, showing success rates between 63.5 and $90 \%$ cases $[6,8]$ if endoscopy was performed early. However, some authors report a preference for pushing the FB into the stomach, expecting it to be digested, reporting a low withdrawal rate (27\%) [21].

Objects located in the esophagus were appropriately removed by endoscopy in 12 dogs (63\%), a success rate similar to that described in previous studies [12]. The FB was moved into the stomach in three animals in the present study, a procedure that has been highlighted by some authors in previous studies [12,21]. Although removal of esophageal FBs is considered an appropriate therapeutic possibility, movement of these structures is not always possible because there are adherences or perforations in the organ that prevent this. Faced with the impossibility of completely removing the FB from this organ, pushing it into the gastric lumen is suggested, leading the patient to undergo an open surgical intervention, gastrotomy instead of thoracic esophagotomy, as long as the case in question allows. Thoracic access to the esophagus, in addition to being a more elaborate technique than access to the stomach, may produce greater surgical morbidity and the possibility of worsening the patient's prognosis, since esophageal healing occurs 


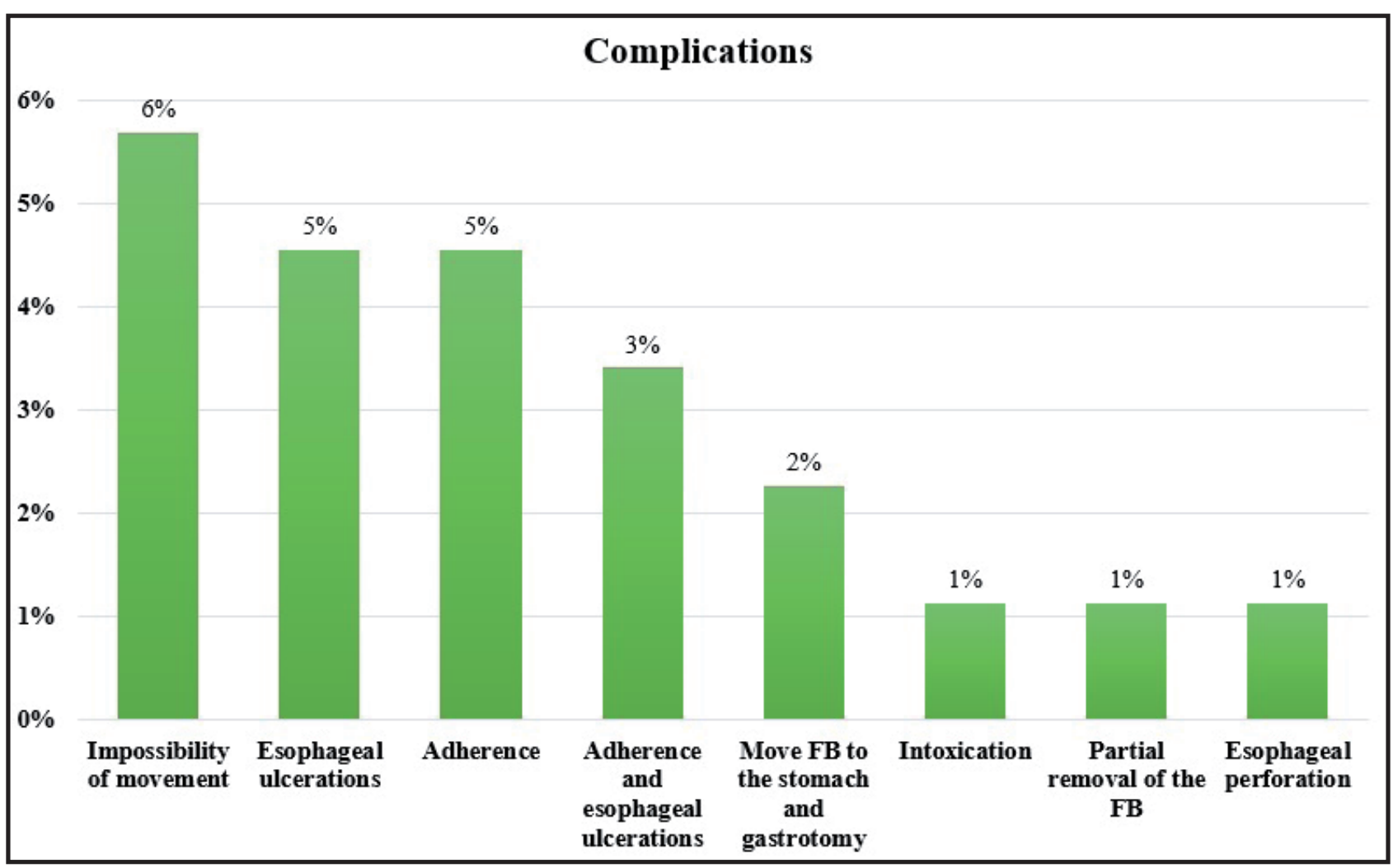

Graph 3. Complications presented during the endoscopic procedure for esophageal and gastric FB removal in 88 dogs between 2013 and 2019.

more poorly due to the absence of serosa, peristalsis, the impossibility of omentalization and the segmental irrigation of the organ [17]. Thus, the choice of upper digestive endoscopy in the diagnosis of esophageal FB is essential both in the attempt to remove the object completely and also to direct it to the stomach.

The main points of esophageal stenosis are in the region of the upper esophageal sphincter, chest entrance, base of the heart and near the region of the gastroesophageal junction [20]. We observed that most esophageal FBs were in the region of the esophageal hiatus $(53 \%)$, corroborating what was described by other authors [6], but differing from another study in which $68.2 \%$ of FBs were found caudal to the base of the heart [12].

A prolonged time between the observation of clinical signs by the owner and the endoscopic procedure occurred with many patients in this study, which makes it possible to infer that the distal location of the FB in the esophageal lumen was a result of chronicity of the case and organ peristalsis. If there were early diagnosis and endoscopic intervention, it would be possible to find these objects in portions more cranial to the gastroesophageal junction. In addition, early diagnosis and treatment of these conditions would also influence the reduction of esophageal wall ischemic processes due to FB and, consequently, reduce complications of ulcerations evaluated.

Regarding the FBs found, the results of the present study corroborate previously described results, in which animal bones as FBs had a high prevalence $[6,12]$. Fruit pits represented a high percentage in the present study (10\%), especially mango (7\%), unlike in previous studies $[6,12,21]$. According to the Companhia Urbanizadora da Nova Capital do Brasil (Novacap), there were on average of 500,000 mango trees of different species in the Federal District in 2016 [4]. Besides, it was forecasted that approximately 2000 seedlings would be planted per year, thus explaining the high prevalence of this type of FB in dogs in the region.

Most dogs were of small breeds (55\%), corroborating literature data, which show a prevalence of 61.7 to $73 \%$ of esophageal FBs in small dogs [12,21]. However, some studies have shown that gastrointestinal FBs mostly affected large breeds such as Labrador retriever (13.6\%), Golden retriever (5.3\%) [8], Staffordshire terrier (30\%) and English bull terrier (7\%) [7]. It is important to highlight the high prevalence of Shih tzu (18\%) and Yorkshire (6\%) in the present study, agreeing with the prevalences found in previous studies in which Shih tzu represented between 11 and $18 \%$ and Yorkshire 9\% [12,21]. The West Highland white terrier (WHWT) 
breed, despite having reported gastrointestinal FBs ingestion [11,20], had a low prevalence in this study, representing only $1 \%$ of dogs. This can be explained by the low popularity of the breed in Brazil, where WHWTs are not among the ten most popular breeds in the country, while Shih tzu and Yorkshire occupy second and third place, respectively, following only mixed breed dogs [3]. Regarding the dogs of larger breeds, they were little representative in this study, which included Rotweiller and some UDB dogs, maybe due to the preference of dog owners in our region.

Dogs weighing less than 10 pounds have a higher rate of complications from FB ingestion [6]. It is believed that this relationship can be explained by the size of the FB compared to the size of the animal, i.e., the same FB is potentially more dangerous in smaller dogs. Complications observed in the present study included perforation (one case) and esophageal ulceration (eight cases). In contrast, esophageal perforation was the most common complication in a previous study, occurring in association with pneumomediastinum, a comorbidity not observed in the present study [6].

Young dogs are most commonly diagnosed with FBs and their ingestion should always be a suspect in puppies with acute or chronic vomiting [16]. Regarding age, $66 \%$ of dogs affected were between one and five years old, with most of them (22\%) only one year old. This information is similar to that reported in the literature, where the age range of most dogs diagnosed with gastrointestinal FBs is between 2.5 and 4 years [5-7].

Risk factors associated with the clinical evolution of dogs with esophageal FB have already been established [21]. The main marker is the severity of esophagitis, as this is an important predictor of morbidity and length of hospital stay. It was observed that dogs with clinical signs for more than 19 hours showed severe esophagitis, which resulted in longer hospitalization and greater complications. Esophagitis can range from mild mucosal inflammation to ulceration and transmural involvement [19]. Esophageal FB movement causes repeated swallowing and relaxation of the lower esophageal sphincter. Repeated swallowing creates a refractory period when there is no peristalsis and food remains in the esophagus. Pressure necrosis occurs secondary to continuous peristaltic activity and damage extends from the mucosa to the submucosa. The resulting esophagitis affects the effectiveness of peristaltic activity with reduced cardia tone and subsequent acid reflux [21]. Transmural esophageal necrosis may lead to overflow of food and toxins into the mediastinum and pleural space. Therefore, mediastinitis and pleuritis may cause severe systemic clinical signs [18].

Gastrointestinal tract obstruction results in disturbed water, acid-base and electrolyte balance due to hypersecretion and sequestration, which is exacerbated by vomiting and impaired ingestion of fluids and nutrients [2]. To make the diagnosis of obstruction by $\mathrm{FB}$, it is essential to use imaging resources that help in the clinical confirmation of the existence of esophageal and gastrointestinal obstruction points, and thus establish the best form of treatment. It is well known that simple ultrasound and radiographic evaluations are often performed for this purpose. Despite the advantage of not requiring anesthesia, the identification of changes in the lumen of these organs through these imaging examinations may not be satisfactory, leading to a false negative diagnosis regarding the presence of esophageal obstruction [21]. Compared to radiography and ultrasound, endoscopy has the advantage of making tissue analysis feasible and being curative in cases of gastrointestinal FBs [15].

In one of the study patients, radiographic and ultrasound evaluations did not show the presence of a gastric FB. However, during endoscopic evaluation a bottle cap was found in the gastric body region that could be successfully removed. This finding is reinforced by data from a previous study in which, the FB was not observed with simple radiography in 91 (28.6\%) of 318 dogs [8]. The use of barium for radiographic contrast is contraindicated if endoscopy is a possibility because this contrast impairs visualization [21]. In a study in which 22 dogs underwent radiographic examinations and esophagoscopy, the latter was considered the better diagnostic method [10]. Thus, it is inferred that endoscopy is considered a standard procedure in the identification of FBs in the esophageal and gastric lumen [20].

\section{CONCLUSION}

The results of this work indicated that dogs of small breeds, especially Shih tzu and Yorkshire, were more affected due to their popularity in Brazil. Males were more often affected than females, and the most common age was between 1 and 5 years old, especially 1 year old. The higher number of gastric compared to esophageal FBs was related to the long interval 
between ingestion of the object and veterinary care. Although not the most prevalent FB, the high rate of mango pits can be explained by the vast number of mango trees in the Federal District. The complication rate was low compared to the success rate and these findings reinforce the use of endoscopy as the procedure of choice for the diagnosis and removal of gastric and esophageal FBs.
MANUFACTURERS

${ }^{1}$ Aizu Olympus Co. Ltd. Fukushima, Japan.

${ }^{2}$ Rhosse Instrumentos e Equipamentos Cirúrgicos EIRELI. São

Paulo, SP, Brazil.

${ }_{3}^{3}$ Jiuhong Medical Instrument Co. Ltd. Changzhou, China.

${ }^{4}$ Zscan Software Ltda. Goiânia, GO, Brazil.

Declaration of interest. The authors report no conflict of interest.

\section{REFERENCES}

1 Binvel M., Poujol L., Peyron C., Dunie-Merigot A. \& Bernardin F. 2018. Endoscopic and surgical removal of oesophageal and gastric fishhook foreign bodies in 33 animals. Journal of Small Animal Practice. 59(1): 45-49.

2 Boag A.K., Coe R.J., Martinez T.A. \& Hughes D. 2005. Acid base and electrolyte abnormalities in dogs with gastrointestinal foreign bodies. Journal of Veterinary Internal Medicine. 19(6): 816-821.

3 Doghero. 2018. Censo Canino 2018: top raças e nomes de cachorro. Disponível em:_<https://love.doghero.com.br/ dicas/censo-canino-2018-racas-nomes-de-cachorro-mais-populares $>$. [Accessed online in August 2018].

4 G1.2016. Pés de mangas em ruas do DF equivalem a 44\% da produção do país. Disponível em: <http://g1.globo.com/ distrito-federal/noticia/2016/11/pes-de-mangas-em-ruas-do-df-equivalem-44-da-producao-do-pais.html>. [Accessed online in August 2019].

5 Guilford W.G. 2005. Upper Gastrointestinal Endoscopy. In: McCarthy T.C. (Ed). Veterinary Endoscopy for the Small Animal Practitioner. Saint Louis: Saunders Elsevier, pp.279-321.

6 Gianella P., Pfammatter N.S. \& Burgener I.A. 2009. Oesophageal and gastric endoscopic foreign body removal: complications and follow up of 102 dogs. Journal of Small Animal Practice. 50(12): 649-654.

7 Hayes G. 2009. Gastrointestinal foreign bodies in dogs and cats: a retrospective study of 208 cases. Journal of Small Animal Practice. 50(11): 576-583.

8 Hobday M.M., Pachtinger G.E., Drobatz K.J. \& Syring R.S. 2014. Linear versus non-linear gastrointestinal foreign bodies in 499 dogs: clinical presentation, management and short term outcome. Journal of Small Animal Practice. 55(11): 560-565.

9 Houlton J.E.F., Herrtage M.E., Taylor P.M. \& Watkins S.B. 1985. Thoracic oesophageal foreign bodies in the dog: a review of ninety cases. Journal of Small Animal Practice. 26(9): 521-536.

10 Jankowski M., Spużak J., Kubiak K., Glińska-Suchocka K. \& Nicpoń J. 2013. Oesophageal foreign bodies in dogs. Polish Journal of Veterinary Sciences. 16(3): 571-572.

11 Johnson G.F., Jones B. \& Twedt D.C. 1978. Gastrointestinal fiberoptic endoscopy in small animals. In: Proceedings of the $28^{\text {th }}$ Gaines Symposium (New York, U.S.A.). pp.27-31.

12 Juvet F., Pinilla M., Shiel R.E. \& Mooney C.T. 2010. Oesophageal foreign bodies in dogs: factors affecting success of endoscopic retrieval. Irish Veterinary Journal. 63(3): 163-168.

13 Moore A.H. 2001. Removal of oesophageal foreign bodies in dogs: use of the fluoroscopic method and outcome. Journal of Small Animal Practice. 42(5): 227-230.

14 Moore L.E. 2003. The advantages and disadvantages of endoscopy. Clinical Techniques in Small Animal Practice. 18(4): 250-253.

15 Mourya A., Mehta H.K., Gupta D.K., Singh B., Tiwari A., Shukla P.C. Sheikh A.A. \& Bhagat R. 2018. Gastrointestinal Fiberscopy in Dogs: A review. Journal of Entomology and Zoology Studies. 6(2): 2330-2335.

16 Pratt C.L., Reineke E.L. \& Drobatz K.J. 2014. Sewing needle foreign body ingestion in dogs and cats: 65 cases (2000-2012). Journal of the American Veterinary Medical Association. 245(3): 302-308.

17 Radlinsky M.G. 2013. Surgery of the Digestive System. In: Fossum T.W. (Ed). Small Animal Surgery. 4th edn. Saint Louis: Mosby Elsevier, pp.428-437.

18 Rousseau A., Prittie J., Broussard J.D., Fox P.R. \& Hoskinson J. 2007. Incidence and characterization of esophagitis following esophageal foreign body removal in dogs: 60 cases (1999-2003). Journal of Veterinary Emergency and Critical Care. 17(2): 159-163.

19 Tams T.R. 2005. Gastroenterologia de Pequenos Animais. 2.ed. São Paulo: Roca, 472p. 
F.M. Poggiani, R.P.C. Duarte, M.I.S. Santana \& P.D. Galera. 2020. Endoscopic Removal of Foreign Body in Upper Gastrointestinal Tract in Dogs: Success Rate and Complications. Acta Scientiae Veterinariae. 48: 1735.

20 Tams T.R., Rawlings C.A. 2011. Small Animal Endoscopy. 3rd edn. Saint Louis: Mosby Elsevier, 682p.

21 Thompson H.C., Cortes Y., Gannon K., Bailey D. \& Freer S. 2012. Esophageal foreign bodies in dogs: 34 cases (2004-2009). Journal of Veterinary Emergency and Critical Care. 22(2): 253-261.

22 Zoran D.L. 2001. Gastroduodenoscopy in the dog and cat. The Veterinary Clinics of North America. Small Animal Practice. 31(4): 631-656. 